Rentor Y. Cafino, MD

Department of Ears, Nose, Throat Head and Neck Surgery

Zamboanga City Medical Center
Correspondence: Dr. Rentor Y. Cafino Department of Ears, Nose, Throat - Head and Neck Surgery Zamboanga City Medical Center, Outpatient Bldg, Dr. D. Evangelista St., Sta. Catalina, Zamboanga City 7000 Philippines

Phone: +639173015910

Email: rcafino@gmail.com

The author declared that this presents original material that is not being considered for publication or has not been published or accepted for publication elsewhere or in part, in print or electronic media; that the requirements for authorship have been met by the author, and the author believes that the manuscript represents honest work.

Disclosures: The author signed a disclosure that there are no financial or other (including personal) relationship, intellectual passion, political or religious beliefs, and institutional affiliations that might led to conflict of interest.

Presented at the Philippine Society of Otolaryngology Head and Neck Surgery 1st virtual Surgical Innovation Contest (1st Place) November 25, 2020.

\section{The Use of Commercially Available Non-Medical Grade USB Cameras for Physician Guided ENT Out-Patient Self-Examination During the COVID-19 Pandemic}

\begin{abstract}
Objective: To describe the use of commercially available, non-medical grade USB cameras in a non-contact examination of simple cases involving the ears, nose and oral cavity of patients during the COVID-19 pandemic.
\end{abstract}

\section{Methods:}

Design: Instrument innovation

Setting: $\quad$ Tertiary Government Training Hospital

Participants: Patients who consulted at the ENT-HNS outpatient department during the COVID-19 pandemic.

Results: Commercially available USB cameras were able to provide basic visualization of the ears, nose and oral cavity. The non-medical grade USB cameras captured lower quality images when compared to the medical grade endoscopes but provided enough visualization to aid in the examination and diagnosis of simple cases. There was a learning curve in using the set-up but patients were able to adjust well, taking an average time of 2.7 minutes to complete the examination.

Conclusion: Non-medical grade USB cameras may play a role in aiding otolaryngologists in examining simple cases during the COVID-19 pandemic. Integration of this system into current examination practices may offer an extra layer of protection for otolaryngologists and patients alike. However, the use of these instruments as part of regular ENT practice may be controversial and will need further study.

Keywords: USB camera; no-contact examination; virtual physical examination; Onsite Virtual Examination (OnVEx); COVID-19

The COVID-19 pandemic has changed the face of the medical world as we know it. Because many health care professionals around the world were infected while providing care to their patients, health care institutions turned to telemedicine to avoid the high risk of infection. 


\section{SURGICAL INNOVATIONS AND INSTRUMENTATION}

Although telemedicine with thorough history taking and augmented patient self-examination may aid the physician in making a diagnosis, this is often not enough. In particular, otolaryngology patients need to consult or are referred to have their orifices visualized in person. ${ }^{1}$ The majority of outpatient otolaryngology consults involve such ear diseases as otitis media, externa and impacted cerumen followed by nasal stuffiness and throat pain., ${ }^{2,3}$ These diseases are difficult to diagnose with clinical history alone, and treatment depends on physical examination findings, necessitating visualization. This places otolaryngologists in a unique and risky position.

Extra precautions taken when attending to patients who are physically present during the pandemic include screening, appropriate personal protective equipment (PPE), proper distancing, and standard disinfection protocols. ${ }^{4}$ Despite the availability of protocols and recommendations, hospitals are faced with PPE and supply shortages, ${ }^{5,6}$ forcing health care providers to adapt, some for better and some for worse. ${ }^{7,8}$ In adapting to the changing health situation, the use of unconventional methods may offer temporary solutions.

We describe one such attempt at adaptation, using commercially available USB cameras for no-contact examinations of the ears, nose, and oral cavity which we termed as Onsite Virtual Examination (OnVEx). This paper also describes their uses and applications in face-to-face otolaryngologic consultations during the COVID-19 pandemic.

\section{METHODS}

\section{Materials}

1. Two pen type USB cameras for the ears and nose and 1 USB camera mounted on the camera handle for the oral cavity (see Figure $1 A, B)$, choosing the USB cameras and fabricating the USB camera handle).

2. A laptop or desktop with a minimum processor of intel i3 (Intel, Sta. Clara, California) running the latest operating system (OS) of choice.

3. A 16-inch generic computer monitor (LED monitor, AOC, Taipei, Taiwan) with compatible connectors (Video Graphics Array or better).

4. A table measuring $1 \times 0.65 \times 0.73(\mathrm{~L} \times \mathrm{W} \times \mathrm{H})$ meters. (Any kind of table can be used as long as the minimum length of 1 meter is met in order to afford 1 meter distancing from the patient. We chose to use a plastic table (Model 280478, LIFETIME, Utah, USA) due to its affordability and portability.

5. Two standard sized armless monobloc chairs (Ruby 1, COFTA Mouldings Corporation, Philippines).

6. Any transparent barrier to separate the examiner from the patient. We chose to use ordinary gauge 8 polyvinyl chloride (PVC) transparent sheet plastic measuring $68 \times 58 \mathrm{~cm}(\mathrm{~W} \times \mathrm{H})$. A frame for the plastic barrier consisted of two 3D printed $C$ clamps printed using PETG in a Prusa i3 Mk3S (Prusa Research, Prague, Czech Republic) and two PVC pipes $2.6 \mathrm{~cm}$ in diameter and $68 \mathrm{~cm}$ in length.

\section{Choosing the USB cameras}

A commercially available pen type USB camera (model i96, unbranded, unspecified manufacturer, China) measuring $15 \mathrm{~cm}$ long with a $5.5 \mathrm{~mm}$ diameter was selected for examination of both the external auditory canal and nasal cavity. (Figure 1A) Although medical grade rigid oto-endoscopes measure $2 \mathrm{~mm}$ in diameter, the average diameter of the external auditory canal is 6 to $8 \mathrm{~mm}^{9,10}$ (theoretically accommodating a $5.5 \mathrm{~mm}$ diameter device), while medical grade rigid nasal endoscopes have a diameter of $4 \mathrm{~mm}$. A shorter $5 \mathrm{~cm}$ USB camera with the same $5.5 \mathrm{~mm}$ diameter (unspecified model, unbranded, unspecified manufacturer, China) was selected for examination of the oral cavity. (Figure 1B) All these USB cameras had 2-meter-long cables. The shorter $5 \mathrm{~cm}$ USB camera was Ingress Protection Code (International Protection Code) IP67 rated, with IP code 6 denoting
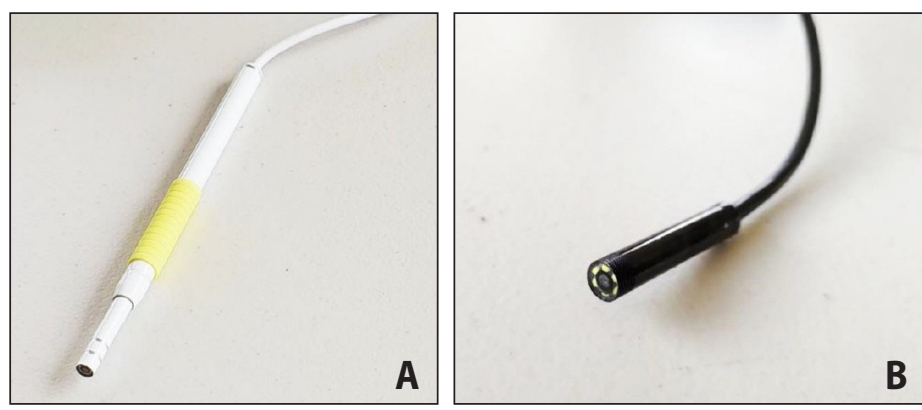

Figure 1. Commercially available USB cameras A. Pen type USB camera for ear and nose examination, B. Short USB camera for oral cavity examination.
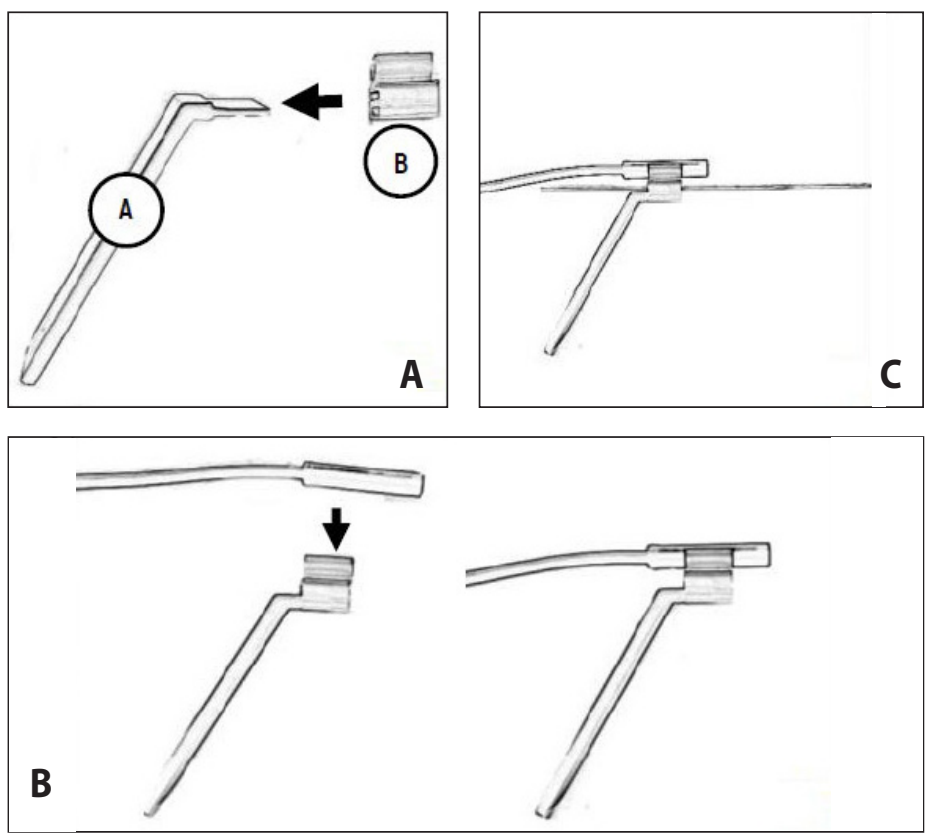

Figure 2. Assembly of USB camera for oral cavity examination A. Camera handle (A) is inserted into the camera-tongue depressor attachment (B); B. The USB camera is mounted onto the camera slot; C. A disposable tongue depressor is inserted on the specified slot. 


\section{SURGICAL INNOVATIONS AND INSTRUMENTATION}

Philippine Journal Of Otolaryngology-Head And Neck Surgery

Vol. 36 No. 1 JANUARY - JUnE 2021

PIOHLS

solid protection from contact with harmful dust, and IP code 7 denoting temporary protection from immersion in water (no specified depth from manufacturer). ${ }^{11}$ The USB cameras selected for the examination of the ear and nose were not waterproof and had no specified IP rating.

\section{Fabricating the USB camera handle}

The USB camera for examining the oral cavity required a handle that could mount the camera on top of a disposable wooden tongue depressor. Inspired by the reusable stainless steel tongue depressor (Weider, PRESICA, Philippines), the handle was modeled in Fusion 360 (Autodesk, San Rafael, California) software for 3D Computer Aided Design (CAD), modeling, manufacturing, industrial design, electronics and mechanical engineering, and $3 \mathrm{D}$ printed using polyethylene terephthalate glycol (PETG) in a Prusa i3 Mk3S (Prusa Research, Prague, Czech Republic).

\section{Assembling the USB camera and handle for Oral Cavity Examination}

After 3D printing the camera handle, part $A$ was attached to part $B$ as illustrated in Figure 2A. The fit had to be snug so no adhesives needed to be applied. The USB camera was then mounted on the slot atop the handle (Figure 2B) and oriented by pointing the camera at an object or image and rotating it until appropriate orientation was achieved. Marking the USB camera with a small piece of tape at the 12-o-clock location helped decrease the time needed to reorient the camera in between patients. Lastly, a wooden tongue depressor was inserted in the specified slot about $2 / 3$ of the way for each use. (Figure $2 C$ ) The author designed the tongue depressor slot to be $2 \mathrm{~mm}$ but observed that some wooden tongue depressors were thinner than others, in such cases, a small piece of paper was inserted into the slot with the tongue depressor to improve the fit.

\section{Setting up the examination space}

The examination table was positioned with a standard monobloc chair on either side in an area with good ventilation. (Figure 3A) The 3D printed C-clamps (Figure 3B) were applied to the middle of the table (Figure $3 C, D$ ) and the PVC pipes were inserted into the slots on the C-clamps. (Figure 3E) A transparent PVC plastic sheet was cut to about $70 \mathrm{~cm}$ and each side taped to the PVC pipe. The pipes were then turned in a clockwise direction until the clear plastic became taut and a final piece of tape was applied to prevent the plastic from unrolling.

One side was designated as the examiner's side and was outfitted with a laptop/desktop computer (Figure 4A) and another computer monitor was placed on the patient's side. (Figure 4B) The computer monitor was attached to the computer on the physician's side via a VGA cable with a USB type $C$ connector. Display settings on the computer were then set to duplicate screen mode. Each USB camera was
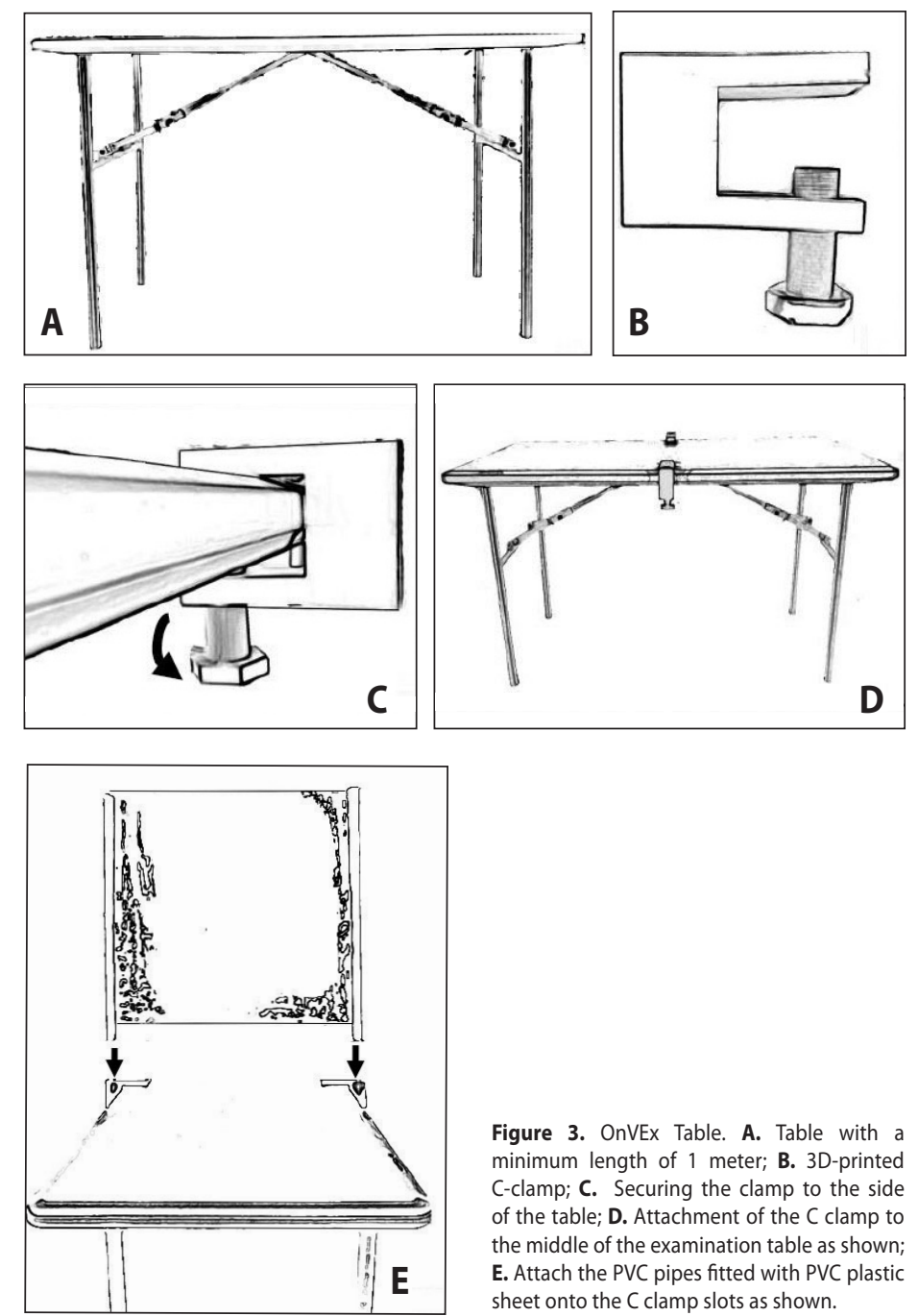

Figure 3. OnVEx Table. A. Table with a minimum length of 1 meter; B. 3D-printed C-clamp; C. Securing the clamp to the side of the table; $\mathbf{D}$. Attachment of the C clamp to the middle of the examination table as shown; E. Attach the PVC pipes fitted with PVC plastic sheet onto the C clamp slots as shown.

separately labeled and laid on a clean linen sheet on the patient's side while its cables were passed underneath the transparent plastic barrier to the examiners side with the USB connectors placed within reach of the physician. The USB connectors were also labeled according to what each camera would be used for. Figure 5 shows the entire Onsite Virtual Examination (OnVEx) physical setup.

\section{Patient criteria}

We pilot tested our OnVEx set up on patients 7 years old and above regardless of gender, who physically consulted or were referred for conditions involving the ears, nose and mouth at the ZCMC ENT-HNS outpatient department from July 2020 to October 2020. All patients had been cleared by the COVID-19 pre-triage, and were considered for pilot testing if they were cooperative and able to follow instructions (or were accompanied by an adult who was able to follow instructions). Hospital employees consulting for routine annual ENT examinations were excluded. 


\section{SURGICAL INNOVATIONS AND INSTRUMENTATION}

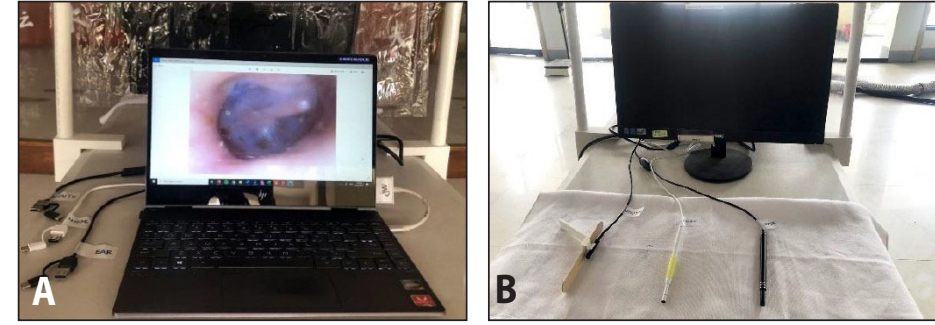

Figure 4. View of examiners and patient's sides; A. Examiner's side; B. Patient's side

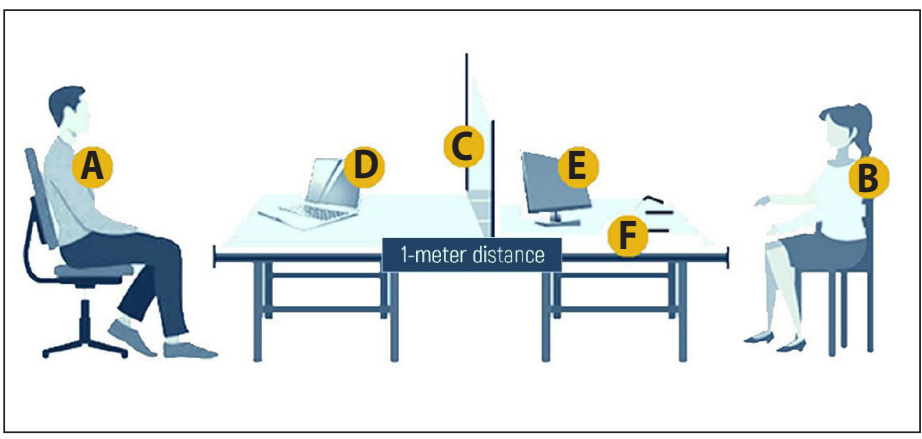

Figure 5. Physical setup for On-Site Virtual Examination (OnVEx). A. Physician; B. Patient; C. Clear plastic barrier; D. Computer/Laptop facing physician; E. Screen facing patient; $\mathbf{F}$. Table measuring at least 1 meter in length.
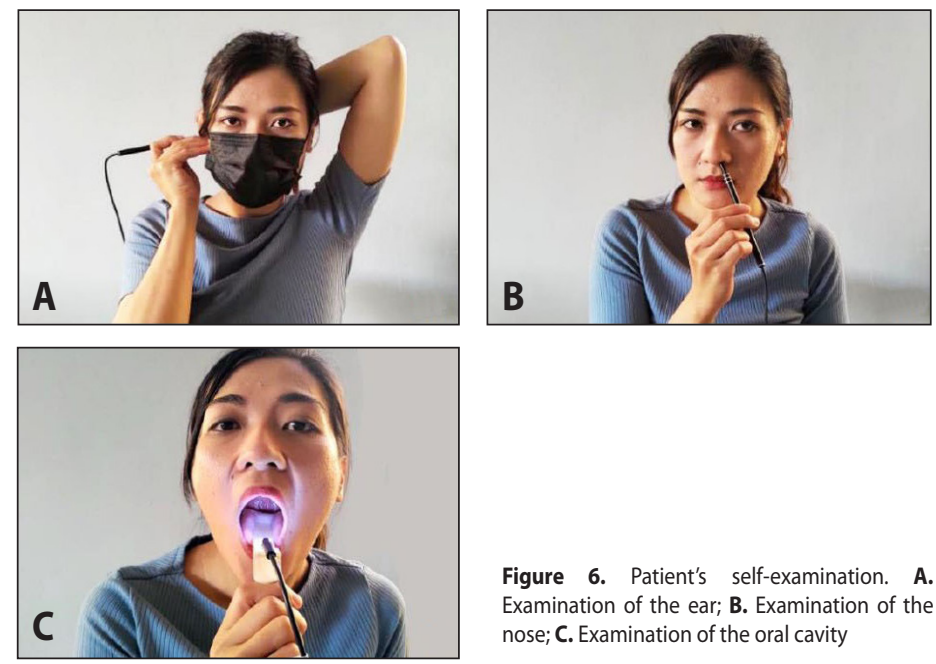

Figure 6. Patient's self-examination. A. Examination of the ear; $\mathbf{B}$. Examination of the nose; $\boldsymbol{C}$. Examination of the oral cavity

\section{Procedure}

1. The patient was seated on the table opposite the examiner and the procedure was explained in the patient's language/dialect or the common language/dialect understood by both the patient and the examiner. The patient was expected to be wearing a mask and was only instructed to remove it during examination of the nose and mouth. The examiner was wearing level I PPE (surgical face mask, alcohol hand wash or spray). Small children were held by an adult companion (carried, or sitting on the lap).

2. A brief and focused history taking was obtained.

3. The USB connector of the camera labeled "ear" was plugged into the computer and the camera program started. The patient was instructed to grasp the camera using the hand opposite to the ear being examined. The patient was then instructed to hold the camera like a pen and orient it by pointing it to his/her face and turn the camera while looking at the screen until proper orientation was achieved. The patient was instructed to pull his/her ear upwards and backwards using his/her free hand (backwards and downwards for pediatric population). Following this, the camera was then inserted into the external auditory canal (EAC) and the tympanic membrane visualized and examined. (Figure 6A) The procedure was then repeated on the opposite ear. If there was presence of discharge or debris, the patient was instructed to clean the ear by gently inserting a cotton pledget and swabbing the ear canal to clear the debris. Examination was discontinued if the EAC was too narrow or obstructed with cerumen. Once examination of the ear was performed, the USB was removed from its port.

4. The USB connector of the camera labeled "nose" was then plugged into the computer and the camera program started. The patient was instructed to hold the camera upright and orient it using the same method described in step 3. Once proper orientation was achieved, the patient was instructed to insert the camera into the nostril opposite the hand holding the camera pointing slightly upwards and towards the midline at about a $15^{\circ}$ angle. (Figure $6 B$ ) The camera was advanced into the nasal cavity until the inferior turbinate, middle turbinate and septum was visualized. The procedure was then repeated on the other side. Once examination of the nose was performed, the USB was removed from its port.

5. The USB connector of the camera labeled "mouth" was plugged next into the computer and the camera program started. The patient was instructed to hold the camera handle with the camera facing the patient. Proper orientation was already done prior to mounting the camera to the handle, however in cases where the camera was displaced, the patient was instructed to orient the camera using the same method described in step 3. The patient was instructed to open his/her mouth and while keeping the tongue inside, gently depress his/ her tongue using the tongue depressor. (Figure 6C) With the tongue depressor in position, the patient was instructed to say "Ah". After oral cavity was examined, the camera was disconnected.

\section{Disinfection}

After use, all endoscopes were replaced and the area wiped down with 1:100 hypochlorite solution prepared according to $\mathrm{DOH}$ guidelines. ${ }^{12}$ The used endoscopes were then wiped down using the 1:100 hypochlorite solution and the camera used for the oral cavity including its handle was soaked in Bis (3-aminopropyl) dodecylamine, Didecyldimethyl ammonium Chloride (Virusolve + EDS, Amity International Health Care, South Yorkshire, U.K.) disinfectant solution for 15 minutes. The pen type USB cameras were placed lens down in a sterile bottle with about $5 \mathrm{ml}$ of Virusolve + EDS disinfectant solution or 


\section{SURGICAL INNOVATIONS AND INSTRUMENTATION}

Philippine Journal Of Otolaryngology-Head And Neck Surgery

Vol. 36 No. 1 JANUARY - June 2021

PIOHIS

with just enough solution to soak the tips of the camera. After soaking, the USB cameras were wiped down once again and left to air dry.

\section{Examination Flow}

Patients who consulted at the ENT-HNS OPD first passed through the COVID-19 pre-triage area where they were pre-screened by the medical officer in charge. Once cleared, the patients were directed to the outpatient department and triaged by the resident-in-charge. Patients who sought consult for conditions of the ears, nose and oral cavity were then directed towards the OnVEx examination area where history and examination using the described method was performed by three ENT-HNS resident physicians.

\section{Special Interventions}

Patients with ear discharges and cerumen in the ear canal were first asked to perform ear cleaning by gently swabbing the ear canal with a cotton pledget to remove the debris. If the ear canal remained obstructed, the patient was taken to the department's makeshift negative pressure booth where the examination was completed after ear cleaning and suctioning. Patients that required further examination and special ENT procedures were directed to the makeshift negative pressure booth where the procedures were performed and examination carried out.

\section{Documentation and Data Analysis}

Examinations were performed by the author and two other ENT-HNS resident physicians. The procedure was practiced among all 3 residents prior to implementation on patients. However, no formal training was done. Documentation was performed by the junior residents and the results were logged in Google Sheets (Google, California, USA). Photo documentation was obtained by the respective examiner. Data was analyzed using simple sums and frequencies. No evaluation/feedback tool was administered to the patients or physicians. Suggestions for improvement were gathered among the physicians in an informal manner.

\section{RESULTS}

A total of 17 patients ( 7 females and 10 males) were examined using OnVEx during the pilot trial period. There were four children (ages 7 to 11 years old) and 13 adults (ages 18 to 63 years old). Age distribution was skewed to the left with a median age of 22 and an interquartile range of 12. Three ENT-HNS physicians including the author performed the examinations during the study period. All three residents were adept at performing a basic ENT examination and had tested the OnVEx system prior to examining patients.

All patients examined during this time had ear complaints and the most common diagnosis was otitis externa. Further examination of the

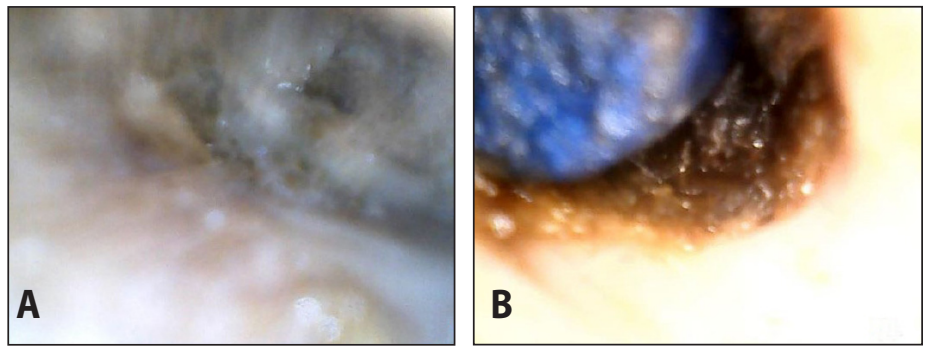

Figure 7. Overtly abnormal ear findings seen on ear examination. A. Picture of a patient's ear canal with otomycosis taken using a USB camera; B. Picture of a patient's ear canal with a foreign body taken using a USB camera.
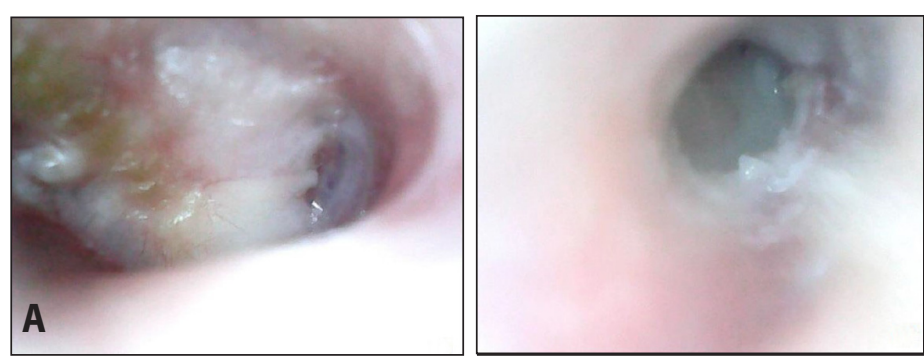

Figure 8. Patient with bilateral cholesteatoma. A. Right tympanic membrane with whitish debris behind the tympanic membrane and a suspected attic retraction pocket; $\mathbf{B}$. Left tympanic membrane with a near total perforation and whitish debris.
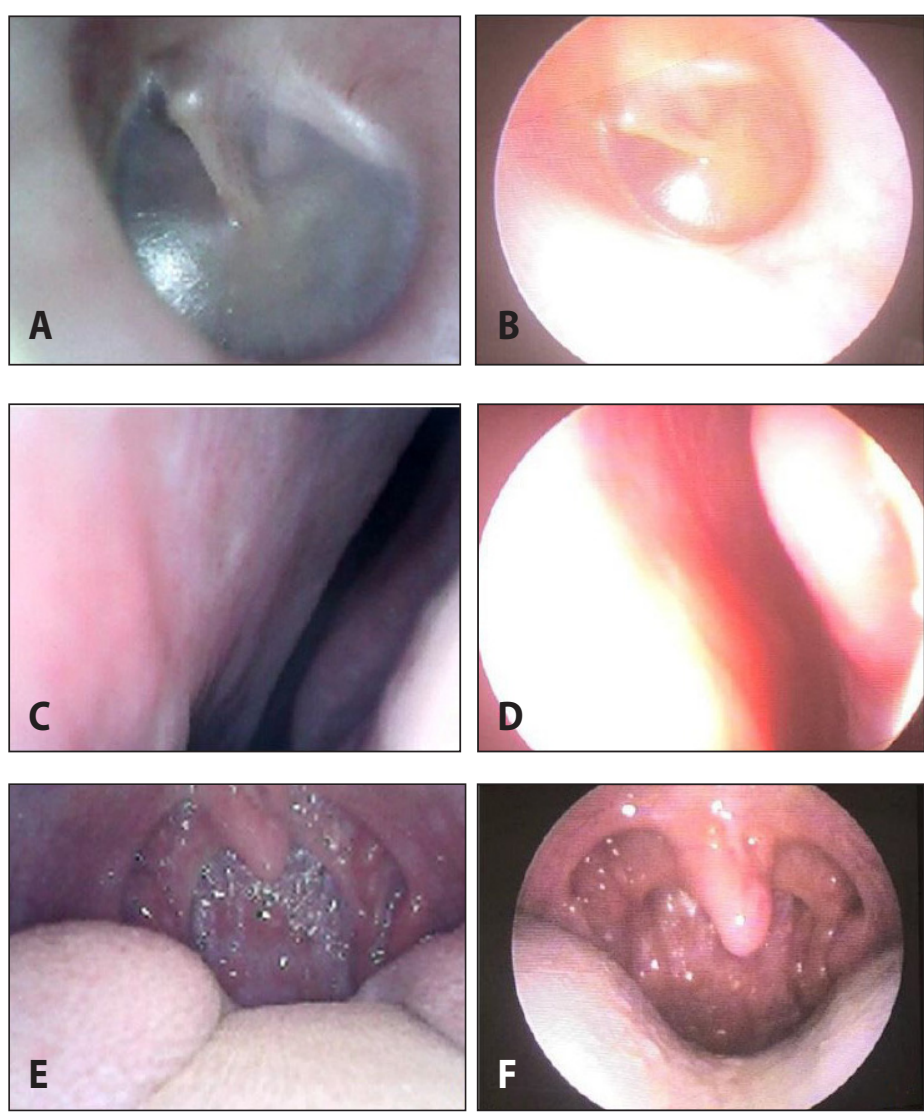

Figure 9. Comparison of photos taken by non-medical grade endoscopes (left) and those taken by a Storz endoscope system (right) 


\section{SURGICAL INNOVATIONS AND INSTRUMENTATION}
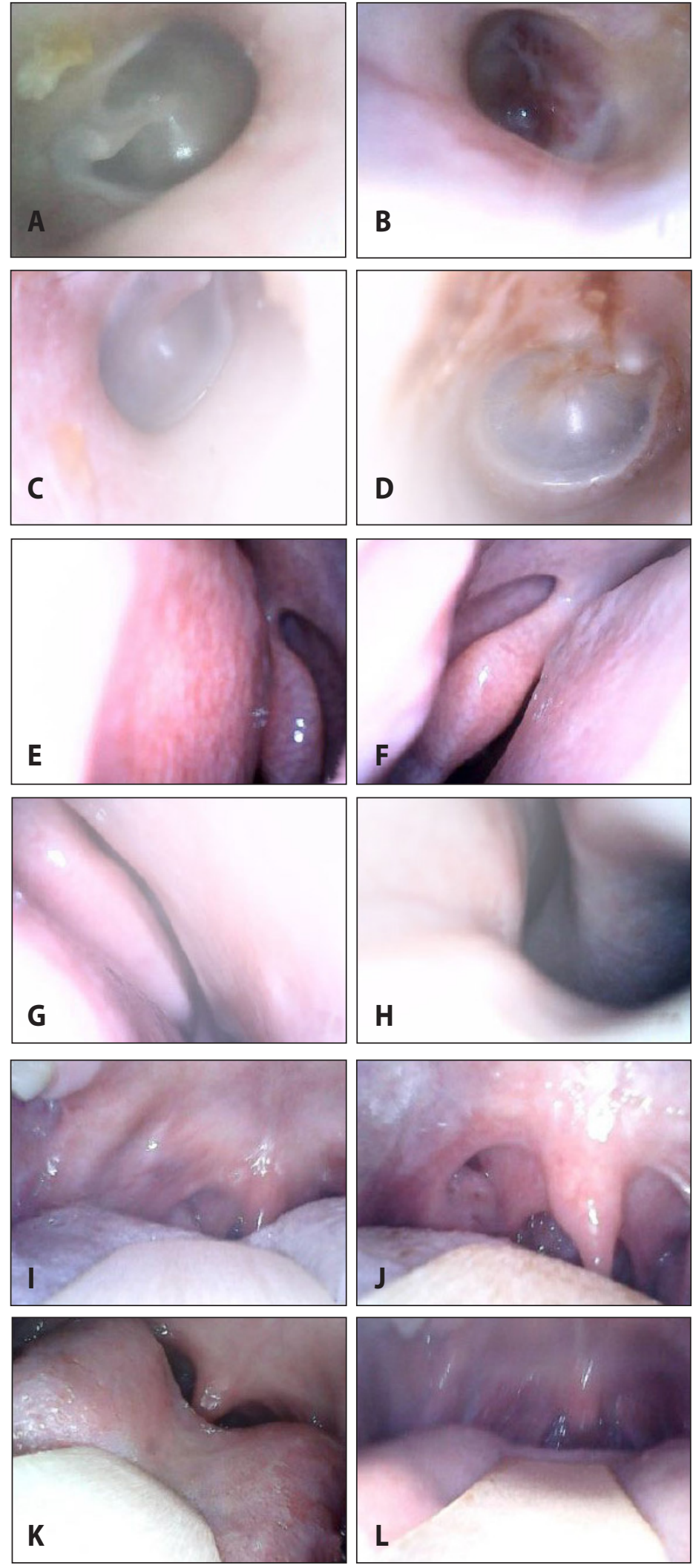

Figure 10. Compilation of images taken during OnVEx showing differences in exposure, focus and orientation. nose and oral cavity showed normal results with an average examination time of 2.7 minutes. Two patients had to undergo additional procedures in the makeshift negative pressure booth, one had otomycosis (Figure $7 A$ ) and underwent ear cleaning while the other had a retained foreign body (rubber) (Figure 7B) in his left ear. One patient was diagnosed as bilateral chronic suppurative otitis media with cholesteatoma (Figure $8 A, B)$ and was admitted for further evaluation and management.

Images captured using the USB cameras were of a significantly lower quality compared to that of a medical grade camera. Figure 9 shows a side-by-side comparison of photos taken by non-medical grade endoscopes and those taken by a Storz endoscope system (Telecam C-mount one chip camera head NTSC, Storz, Tuttinlingen, Germany). At the time of documentation, the author had no access to a digital video recorder for the Storz system and the screen of the endoscope tower was photographed using a mobile phone camera (iPhone 10, Apple, CA, USA). The figures on the left were taken using the USB cameras while those on the right were taken using the Storz Telecam documentation system. The pictures on the right showed paler mucosa than their counterparts. The images on the right were sharper and better illuminated.

\section{DISCUSSION}

Endoscopic visualization is usually part of the otolaryngology examination battery and is performed by a trained operator with specialized endoscopes. However, due to the COVID-19 pandemic, most of these procedures place the examiner at risk and would require the physician to be in full Personal Protective Equipment (PPE) in order to mitigate the risk. ${ }^{13,14}$ Due to the shortage of PPEs caused by the pandemic, their use has been reserved for limited situations. ${ }^{14}$ Endoscopic visualization performed by patients on themselves under supervision of an otolaryngologist at a safe distance could theoretically allow examination without close physical contact. However, medical grade endoscopes are costly and require specialized training. We used commercially-available USB cameras with dimensions similar to endoscopes used in ENT examinations in place of the medical grade endoscopes. The cameras are relatively inexpensive and can be handled by an untrained person. Melder and Mair ${ }^{15}$ compared a digital camera to the traditional $35 \mathrm{~mm}$ lens system in endoscopic photography and found that images taken using a digital camera were comparable and sometimes even superior to the traditional counterpart. The aforementioned USB cameras use the same digital camera system but have lower resolution due to their size, explaining the lower image quality when compared to a medical grade endoscopy system.

Endoscopic examination performed by a trained individual can visualize the intended structures in standardized fashion with full focus and proper orientation. However, if performed by an untrained individual, visualization will be expected to vary. Examples of such 


\section{SURGICAL INNOVATIONS AND INSTRUMENTATION}

Philippine Journal Of Otolaryngology-Head And Neck Surgery

VOL. 36 No. 1 JANUARY - JUNE 2021

images are shown in Figure 10. These images were taken after a maximum of 5 attempts to instruct the patient. Some pictures were well focused and centered ( $A, D, E, J$ and $L$ ) while others were poorly focused and not well-oriented ( $B, C, F, G, H$ and $K)$. Despite these limitations, examination of the intended structures was still possible. Despite the lower image quality and occasional poor orientation, the examiners were able to diagnose simple cases.

Examination of the ears, nose, and throat using medical grade endoscopes through a small hole in a mask or a mask with an endoscopy valve may lessen aerosolization during routine ENT examination ${ }^{15}$ but this still requires the examiner to be in close contact with the patient (less than 1 meter). OnVEx has the advantage of a no-touch setup and physical distancing of 1 meter separated by a physical barrier which could theoretically allow for use of level I PPE. ${ }^{14,16}$ However, unlike the the previously mentioned methods, OnVEx requires a wide open space which may not be feasible in small clinics, and procedures such as removal of foreign bodies and laryngoscopy cannot be performed.

The makeshift negative pressure booth inspired by the Korean walk in swab booths ${ }^{17}$ was designed to allow otolaryngologists to perform aerosol generating procedures (AGP) safely but was used to perform a full ENT examination as well. Compared to OnVEx, preparation time took longer ( 1 minute versus 5 minutes) and less resources was required (e.g. cleaning solution, PPE).

This pilot trial had other limitations. Due to the pandemic, the trial sample was limited and the cases seen were not varied. Because the trial was performed during the early stages of the COVID-19 pandemic, extreme caution was implemented thus limiting physician - patient interaction to only the bare essentials. Documentation of the patients educational status, literacy, language/dialect spoken, and experience feedback was not complete, and we cannot speculate on any associations between these variables and the outcomes of the OnVEx examination. Should a formal clinical trial of this innovation be performed, the author recommends including a larger and more varied patient population and investigation of other factors that may affect the outcome of the OnVEx examination.

This initial experience demonstrated that non-medical grade USB cameras when integrated into a physician guided self-examination by patients was able to provide basic visualization of the ears, nose, and oral cavity. Although the image quality and orientation were not as good as endoscopic documentation performed by the examiner using a medical grade endoscope, visualization using USB cameras aided the examiners in making a diagnosis without having to come in close contact with the patient. Non-medical grade USB cameras may play a role in the examination of the ENT patient. Simple complaints such as ear pain, nasal stuffiness, and dysphagia may be initially assessed by means of no-contact examination using the described camera system. Although there was a learning curve to using the USB cameras, patients were generally able to adjust well. Integration of this system into current examination practices may provide an extra layer of protection for the otolaryngologist and patient alike during the COVID-19 pandemic. The use of these instruments as part of the regular ENT practice however may be controversial and will need further study.

\section{ACKNOWLEDGEMENTS}

I wish to thank our department chairman Dr. Justin Elfred Lan B. Paber for advice on patient examination flow, optimizing physical setup and coining the term OnVEx, our Research Coordinato Dr. Nurhada A. Israel for guiding me in writing this paper, and my co-resident physicians Dr. Maria Monique Theresita Soliven for serving as an examiner and providing photographs and line drawings, and Dr. Audrey Ann Cortez for serving as an examiner for OnVEx.

\section{REFERENCES}

1. McCoul ED. Grasping what we cannot touch: examining the telemedicine patient. JAMA Otolaryngol Head Neck Surg. 2020 Jun 18;146(8):685-686. DOI: 10.1001/jamaoto.2020.1266; PubMed PMID: 32556313.

2. Fasunla AJ, Samdi M, Nwaorgu OG. An audit of ear, nose and throat diseases in a tertiary health institution in South-western Nigeria. Pan Afr Med J. 2013 Jan;14(1) DOI: 10.11604/ pamj.2013.14.1.1092 PubMed PMID: 23503692 PubMed Central PMCID: PMC3597854.

3. Zamboanga City Medical Center Department of ENT-HNS Annual Census 2019. Unpublished data.

4. PSO-HNS COVID-19 Advisory No. 5: Recommendations on the OPD workflow during the COVID-19 pandemic, 04 May 2020. [Internet] [cited 2020 June 16] Available from: https:// pso-hns.org/2020/05/04/advisory-no-5-recommendations-on-the-opd-workflow-during-thecovid-19-pandemic/.

5. Park CY, Kim K, Roth S, Beck S, Kang JW, Tayag MC, Griffin M. Global Shortage of Personal Protective Equipment amid COVID-19: Supply Chains, Bottlenecks, and Policy Implications. Asian Development Bank Briefs. 2020 April (130)

6. Duque F III. Department Circular 2020-0175 (Republic of the Philippines Department of Health, Office of the Secretary). Manila: Department of Health. 2020. pp. 1-3.

7. Press C. Coronavirus: The NHS workers wearing bin bags as protection. [Internet] BBC News. April 5 2020. Available from: https://www.bbc.com/news/health-52145140.

8. Armijo PR, Markin NW, Nguyen S, Ho DH, Horseman TH, Lisco, SJ, Schiller AM. 3D printing of face shields to meet the immediate need for PPE in an anesthesiology department during the COVID-19 pandemic. American Journal of Infection Control. 2021(49): 302-308.

9. Yu JF, Lee KC, Wang RH, Chen YS, Fan CC, Peng YC, et al. Anthropometry of external auditory canal by non-contactable measurement. Appl Ergon. 2015 Sep;50:50-5. DOI: 10.1016/j. apergo.2015.01.008; PubMed PMID: 25959317

10. Graceck RR. Anatomy of the Auditory and Vestibular System. In : Snow JB, Wackym PA, Balenger $\mathrm{JJ}$, editor. Ballenger's Otolaryngology: Head and Neck Surgery 17th Ed. Shelton: London; PMPH USA. 2009. P. 62-66.

11. Ingress Protection (IP) Ratings. 2013. [cited 2021 March 30] Available from https://www.iec.ch/ ip-ratings.

12. Department Memorandum 0157, Guidelines on Cleaning and Disinfection. Republic of the Philippines Department of Health; 2020. Available from: https://doh.gov.ph/sites/default/files/ health-update/dm2020-0157.pdf.

13. Mick P, Murphy R. Aerosol-generating otolaryngology procedures and the need for enhanced PPE during the COVID-19 pandemic: a literature review. Journal of Otolaryngology - Head and Neck Surgery (2020) 49:29.

14. Dofitas RB, Say AS, Lahoz ER, De Leon JRC, Inso RS, Aison DS, et al. Recommendations for the rational and effective use of personal protective equipment: guidelines for the extended use, re-use, and acceptable reprocessingmethods. [Internet]. Philippine College of Surgeons. 2020 [cited 2020 May 13]. Available from: http://pcs.org.ph/assets/images/PCS-COVID-10 Rationale-Use-of-PPE.pdf.

15. Melder PC, Mair EA. Endoscopic photography: digital or $35 \mathrm{~mm}$ ?. Arch Otolaryngol Head Neck Surg. 2003 May;129(5):570-5. DOI: 10.1001/archotol.129.5.570; PubMed PMID: 12759272.

16. Lapeña JF, Abes FL, Gomez MA, Villafuerte CV, Roldan R, Fullante PB, et al. Otorhinolaryngology out-patient practice in the "Post"-COVID-19 Era: Ensuring a balance between service and safety. Philipp J Otolaryngol Head Neck Surg, 2020; 35(1): 6-29. DOl:10.32412/pjohns.v35i1.1249.

17. Kim SI, Lee JY. Walk-Through Screening Center for COVID-19: An Accessible and Efficient Screening System in a Pandemic Situation. J Korean Med Sci. 2020 Apr 20;35(15):e154 https:// doi.org/10.3346/jkms.2020.35.e154 elSSN 1598-6357.pISSN 1011-8934. 